\title{
Complications after craniosynostosis surgery: comparison of the 2012 Kids' Inpatient Database and Pediatric NSQIP Database
}

\author{
Yimo Lin, MD, ${ }^{1}$ I-Wen Pan, PhD, ${ }^{2}$ Rory R. Mayer, MD, ${ }^{2}$ and Sandi Lam, MD, MBA ${ }^{2}$ \\ 1Department of Neurosurgery, Oregon Health \& Science University, Portland, Oregon; and 2Department of Neurosurgery, \\ Baylor College of Medicine/Texas Children's Hospital, Houston, Texas
}

\begin{abstract}
OBJECT Research conducted using large administrative data sets has increased in recent decades, but reports on the fidelity and reliability of such data have been mixed. The goal of this project was to compare data from a large, administrative claims data set with a quality improvement registry in order to ascertain similarities and differences in content.
\end{abstract}

METHODS Data on children younger than 12 months with nonsyndromic craniosynostosis who underwent surgery in 2012 were queried in both the Kids' Inpatient Database (KID) and the American College of Surgeons Pediatric National Surgical Quality Improvement Program (Peds NSQIP). Data from published clinical craniosynostosis surgery series are reported for comparison.

RESULTS Among patients younger than 12 months of age, a total of 1765 admissions were identified in KID and 391 in Peds NSQIP in 2012. Only nonsyndromic patients were included. The mean length of stay was 3.2 days in KID and 4 days in Peds NSQIP. The rates of cardiac events $(0.5 \%$ in KID, $0.3 \%$ in Peds NSQIP, and $0.4 \%-2.2 \%$ in the literature), stroke/intracranial bleeds ( $0.4 \%$ in KID, $0.5 \%$ in Peds NSQIP, and $0.3 \%-1.2 \%$ in the literature), infection ( $0.2 \%$ in $\mathrm{KID}$, $0.8 \%$ in Peds NSQIP, and $0 \%-8 \%$ in the literature), wound disruption $(0.2 \%$ in KID, $0.5 \%$ in Peds NSQIP, $0 \%-4 \%$ in the literature), and seizures $(0.7 \%$ in KID, $0.8 \%$ in Peds NSQIP, $0 \%-0.8 \%$ in the literature) were low and similar between the 2 data sets. The reported rates of blood transfusion (36\% in KID, $64 \%$ in Peds NSQIP, and $1.7 \%-100 \%$ in the literature) varied between the 2 data sets.

CONCLUSIONS Both the KID and Peds NSQIP databases provide large samples of surgical patients, with more cases reported in KID. The rates of complications studied were similar between the 2 data sets, with the exception of blood transfusion events where the retrospective chart review process of Peds NSQIP captured almost double the rate reported in KID.

http://thejns.org/doi/abs/10.3171/2015.9.FOCUS15383

KEY WORDS craniosynostosis; craniofacial surgery; Kids' Inpatient Database; Pediatric NSQIP; administrative data

$\mathrm{R}$ ESEARCH conducted using large administrative data sets has been increasing over recent decades. ${ }^{25}$ These data sources have the advantages of large sample sizes and wide geographic ranges, but they also have weaknesses including limited granularity of clinical detail and challenges with confounders. The Kids' Inpatient Database (KID; https://www.hcup-us.ahrq. gov/kidoverview.jsp) contains information on inpatient hospitalization discharges. Clinical and resource use information are available from discharge abstracts created by hospitals for billing purposes, and the diagnostic and procedural data are stored as ICD-9 codes. This database includes data from over 4000 hospitals and is nationally representative. The Pediatric National Surgical Quality Improvement Program (Peds NSQIP) is a clinical registry organized by the American College of Surgeons/American Pediatric Surgical Association. At participating hospitals, medical chart review is conducted by trained surgical clinical reviewers who collect perioperative and 30-day postoperative data on randomly assigned patients in certain case categories. The strengths of this data set include the nature of the chart review process and 30-day follow-up, which

ABBREVIATIONS CPT = Common Procedural Terminology; HCUP = Healthcare Cost and Utilization Project; KID = Kids' Inpatient Database; LOS = length of stay; Peds NSQIP = Pediatric National Surgical Quality Improvement Program; PCCG = Pediatric Craniofacial Collaborative Group; PCSPR = Perioperative Craniofacial Surgery Prospective Registry.

SUBMITTED August 2, 2015. ACCEPTED September 29, 2015.

INCLUDE WHEN CITING DOI: 10.3171/2015.9.FOCUS15383. 
result in higher quality data and less reliance on billing code data used by large administrative data sets such as KID. The participating hospitals in the Peds NSQIP are not considered nationally representative, which may limit external validity more than generalizability.

Craniosynostosis is a condition involving the premature fusion of 1 or more cranial sutures, which affects an estimated 1 in 2000-2500 live births worldwide. ${ }^{12,23}$ This condition occurs in isolation in the large majority of cases, but may also occur in association with more than 130 different syndromes. Management is surgical, with procedure recommendations dependent on patient age, surgeon practice, and other clinical factors. ${ }^{11,23}$ The reported rates of perioperative complications after craniosynostosis surgery vary. Published accounts often examine heterogeneous cohorts of patients across multiple years or epochs. ${ }^{10,17,19,24}$ An advantage of research that uses large, national databases, such as KID or Peds NSQIP, is the ability to analyze homogeneous definitions of relatively sizeable patient cohorts in a narrow time window, as surgical techniques evolve quickly. To date, no study has analyzed the rates of complications after craniosynostosis surgery in this manner.

This study reports the rate of perioperative complications after craniosynostosis surgery in nonsyndromic patients younger than 12 months in a 1-year time period (2012) from 2 data sources - a large administrative claims database (KID) and a clinical registry (Peds NSQIP) and compares them to each other and to the complication rates reported in the literature. The goals of this study were 2-fold: 1) to ascertain the rate of perioperative complications after craniosynostosis surgery in the study year; and 2) to examine a large administrative claims data set in comparison with a surgical quality improvement database.

\section{Methods}

\section{Data Source and Statistical Analysis: Peds NSQIP}

Data were gathered from the 2012 reports included in Peds NSQIP, a prospectively collected database organized by the American College of Surgeons and American Pediatric Surgical Association (https://www.facs.org/qualityprograms/acs-nsqip/about). At each participating institution, a trained records reviewer collects surgical cases on an 8-day cycle, and the associated medical records are reviewed for preoperative, perioperative, and postoperative details up to 30 days following the index surgery. Details beyond 30 days are not available in this data set. Surgeries were identified using Common Procedural Terminology (CPT) codes: 61550 (surgery for single cranial suture synostosis) and 61552, 61556, 61557, 61558, 61559, 21175, and 21180 (surgery for multiple sutures and/or other). Preoperative characteristics and periprocedural events up to the 30-day outcomes were collected in Peds NSQIP. History of previous craniosynostosis surgery is unknown. The end points of interest in this study are perioperative complication events. The records from 2012 were selected from patients who were younger than 12 months at the time of admission. To restrict the data set to nonsyndromic cases of craniosynostosis, patients with ICD-9-CM diagnosis codes for concomitant congenital malformations were excluded, including all codes between 740.0 and 759.9 (except 756.0), as well as between 524.0 and 524.9 (dentofacial anomalies).

\section{Data Source and Statistical Analysis: KID}

Data were also gathered from KID (https://www.hcupus.ahrq.gov/kidoverview.jsp), which is one of a family of administrative databases developed by the Healthcare Cost and Utilization Project (HCUP) and sponsored by the Agency for Healthcare Research and Quality. KID is a sample of discharges from all community, nonrehabilitation hospitals in states participating in HCUP. The target universe includes pediatric discharges (patient age 20 years or younger at admission) from community, nonrehabilitation hospitals in the US. KID contains charge information on all patients, regardless of payer, including persons covered by private insurance, Medicaid, Medicare, and the uninsured. Inpatient stay records in KID include clinical and resource use information that is typically available from discharge abstracts: patient demographics, admission type and source, diagnostic and procedural ICD-9 codes, length of stay, disposition, payer data, and total charges. Systemic random sampling was used to select $10 \%$ of uncomplicated in-hospital births, $80 \%$ of complicated in-hospital births, and $80 \%$ of all other pediatric admissions. To obtain national estimates, patient records were provided with weights using the American Hospital Association universe of nonfederal community hospitals as the standard. Hospital data were stratified by the following 6 characteristics: ownership/control, bedsize, teaching status, rural/urban location, region in the US, and status as a freestanding children's hospital. ${ }^{6}$ The number of participating states was 44 in 2012 with data from 4179 hospitals. The KID database contains information on 3,195,782 pediatric discharges, which was weighted to represent $6,675,222$ national discharges in 2012. The basic unit of analysis is a patient discharge, rather than an individual patient. The records for this study were selected from patients younger than 12 months at the time of admission who had both a diagnosis ICD-9-CM code 756.0 (anomalies of the skull and face bones, including craniosynostosis) and 1 or more of the following ICD-9-CM procedure codes: 02.01 (opening of cranial suture: linear/strip craniectomy), 02.03 (formation of cranial bone flap: repair of skull with flap), 02.04 (bone graft to skull), or 02.06 (other cranial osteoplasty: repair of skull NOS). The use of these parameters to capture craniosynostosis surgery has been previously reported in the literature. ${ }^{11,13}$ To restrict the data set to nonsyndromic cases of craniosynostosis, the same criteria for the ICD-9-CM diagnosis codes were applied to Peds NSQIP and KID (excluding codes for concomitant congenital malformations: 740.0-759.9 [except 756.0] and 524.0-524.9). It is important to note that our unit of analysis is patient discharge, not individual patients. All statistical analyses were performed with Stata 12 (StataCorp).

\section{Results}

The cohort of interest that we defined was as follows: children younger than 12 months with nonsyndromic craniosynostosis who underwent operative repair in 2012. In 
KID, an estimated 1765 hospitalizations were identified using these criteria. One-third of patients were female, and the majority (78\%) were white or Hispanic. The mean length of stay (LOS) was 3.2 days. In Peds NSQIP, 391 patients were identified, the median age at surgery was 6 months, $33 \%$ were female, and $83 \%$ were white or Hispanic. The mean LOS was 4 days (Table 1).

The rates of complications are shown in Table 2. The codes and clinical variables used to identify complications are shown in Table 3. Perioperative events and complications were identified in Peds NSQIP using variables predetermined by NSQIP. ${ }^{15,19}$ To clarify definitions, the accompanying data dictionary and Peds NSQIP surgical clinical reviewers were consulted to ensure uniform interpretation. The corresponding categories of perioperative events and complications were identified in KID using ICD-9 codes. Algorithms in the published literature and the recommendations of the US Food and Drug Administration's MiniSentinel program for identifying health outcomes of interest from administrative and claims data were used (http:// www.mini-sentinel.org/methods/outcome_identification/ default.aspx). ${ }^{3,5,6,11,13,15}$ The rates of cardiac events $(0.5 \%$ in KID, $0.3 \%$ in Peds NSQIP, and $0.4 \%-2.2 \%$ in the literature), stroke/intracranial bleeds $(0.4 \%$ in KID, $0.5 \%$ in Peds NSQIP, and $0.3 \%-1.2 \%$ in the literature), infection $(0.2 \%$ in KID, $0.8 \%$ in Peds NSQIP, and $0 \%-8 \%$ in the literature), wound disruption ( $0.2 \%$ in KID, $0.5 \%$ in Peds NSQIP, and $0 \%-4 \%$ in the literature), and seizures $(0.7 \%$ in KID, $0.8 \%$ in Peds NSQIP, and $0 \%-0.8 \%$ in the literature) were low and similar between the 2 data sets. The rates of blood product transfusion (36\% in KID and 64\% in Peds NSQIP) varied between the 2 data sets. The rates of transfusion in the literature varied between $1.7 \%$ and $100 \% .^{7-9,21,24}$

\section{Discussion}

This study examined the rates of complications after craniosynostosis surgery in nonsyndromic children younger than 12 months in databases from 2012. Data were taken from KID (a national administrative database) and Peds NSQIP (a national quality improvement registry). The rates of most complications were low and similar between the 2 data sets, with the exception of blood transfusion events.

This study analyzes a group of patients using data from the same calendar year. While our cohort definition aims at nonsyndromic single-suture synostosis, the CPT codes are limited in that the specific involved suture and head shape deformities are not defined. In addition, the surgical technique is not known. Taken together, we see a population-based description, but, notably, cannot discern specific case details. The advantages include the large sample size and same time frame from national samples, which minimize variations in practice patterns over time. Large data sets have greater external validity, but are limited in clinical details. In the case of KID data, the large number of participating hospitals represents the majority of the hospitals in the US. The data within these data sets are a nationally representative assessment of the perioperative complication rates that can be expected following the sur- gical management of nonsyndromic craniosynostosis in children younger than 12 months of age.

\section{The Reported Complication Rates After Craniosynostosis Surgery Demonstrated Similarities Between KID and Peds NSQIP}

Previous studies reporting the rates of complications after craniosynostosis surgery are examined for comparison. . $10,14,17,19,21,24$ Traditionally, single-institution reports have limited generalizability. Additionally, clinical series that incorporate decades of data may span significant advancements in surgical techniques, technological development, and paradigm shifts in medical and surgical management. ${ }^{18,20}$ On nearly every data point, there was more variation between individual papers in the literature than there was between KID and Peds NSQIP. For example, the rate of infection was $0.2 \%$ in KID and $0.6 \%$ in Peds NSQIP, but varied between $0 \%$ and $8 \%$ in the published literature. The higher rate in Peds NSQIP may reflect the follow-up period, as the data collected in KID ends at hospital discharge, while Peds NSQIP includes 30-day outcomes.

One important limitation of both KID and Peds NSQIP is the limited follow-up window: KID captures only inhospital events, as this data set represents hospital discharge data. In this case, the discharges are from hospitalizations for the defined index craniosynostosis surgeries. The time period of the data capture per surgical episode is relatively short given that the mean LOS in this cohort is 3.2 days. Complications and other variables are identified based on coded information in KID, with clinical information represented in the form of ICD-9 codes. On the other hand, Peds NSQIP reports events that occur within 30 days after surgery. The complications within this period of time are recorded using medical chart review by institution-specific Peds NSQIP surgical clinical reviewers. In the absence of complete chart documentation, 30-day outcomes include follow-up communication with patients by the surgical clinical reviewers. Any events that occur outside of the delineated time frames for KID and Peds

TABLE 1. Patient characteristics in KID, Peds NSQIP, and the literature

\begin{tabular}{lccc}
\hline Patient Characteristics & KID & Peds NSQIP & Selected Literature* \\
\hline No. & 1765 & 391 & $42-796$ \\
\hline Mean age (mos) & NA & 6.2 & $3-13$ \\
\hline$\%$ female & $32 \%$ & $33 \%$ & $27 \%-46 \%$ \\
\hline$\%$ syndromic & 0 & 0 & $0 \%-20 \%$ \\
\hline Race & & \\
\hline White/Hispanic & $78 \%$ & $83 \%$ & $74 \%-91 \%$ \\
\hline Black & $3 \%$ & $5 \%$ & $5 \%-7 \%$ \\
\hline Other & $7 \%$ & $4 \%$ & $4 \%-5 \%$ \\
\hline$\quad$ Unspecified & $12 \%$ & $8 \%$ & $0 \%-10 \%$ \\
\hline Mean LOS (days) & 3.2 & 4 & $1-7$ \\
\hline Year(s) of data & 2012 & 2012 & $1977-2013$ \\
\hline NA = not available. & \\
* Includes references 4, 7-14, 17-21, 23, and 24.
\end{tabular}


TABLE 2. Complications of nonsyndromic craniosynostosis operations

\begin{tabular}{|c|c|c|c|c|}
\hline Complication & $\mathrm{KID}$ & Peds NSQIP & p Value* & Selected Literature $†$ \\
\hline Infection & $0.2 \%$ & $0.8 \%$ & $<0.001$ & $0 \%-8 \%$ \\
\hline Wound disruption & $0.2 \%$ & $0.5 \%$ & $<0.001$ & $0 \%-4 \%$ \\
\hline Seizures & $0.7 \%$ & $0.8 \%$ & 0.412 & $0 \%-0.8 \%$ \\
\hline Cardiac event & 0.5 & $0.3 \%$ & 0.025 & $0.4 \%-2.2 \%$ \\
\hline Stroke/intracranial bleed & $0.4 \%$ & $0.5 \%$ & 0.291 & $0.3 \%-1.2 \%$ \\
\hline Blood transfusion & $36 \%$ & $64 \%$ & $<0.001$ & $1.7 \%-100 \%$ \\
\hline Sepsis & $1 \%$ & $0 \%$ & $1.000 \ddagger$ & $0.1 \%$ \\
\hline
\end{tabular}

NSQIP would not have been captured by these data. In the case of craniosynostosis surgery, long-term outcomes are essential to judging the success of surgery. Neurocognitive, neuropsychological, aesthetic, and patient/family satisfaction outcomes are notably unavailable in these databases, and currently have no established codes.

\section{The Reported Rates of Complications After Craniosynostosis Surgery Are Low}

We hypothesized that Peds NSQIP would capture more complication events due to the medical chart review process that was conducted by trained clinical reviewers. This process should result in data that are more granular and accurate. In addition, unlike KID, Peds NSQIP allows for some free text entry, which can supplement the level of clinical detail conveyed by the ICD-9 codes. In a comparative study of Peds NSQIP and administrative data, Sellers et al. found that Peds NSQIP demonstrated much higher agreement with the medical charts in comparison with large administrative data. ${ }^{16}$ However, in this study, the rates of complications were very similar between KID and Peds NSQIP. Both databases may underestimate the overall rate of complications for multiple possible reasons, a finding that is reflected in the adult administrative data. ${ }^{3}$ KID data are generated based on billing codes that may either omit certain complications or be unable to capture certain clinical complications due to coding limitations.
Further, as aforementioned, the time frame of event observation only includes single hospitalizations in KID in contrast to the 30-day postoperative time frame for Peds NSQIP. Finally, due to the low incidence of any complications and the sample sizes of each database, it may be difficult to distinguish meaningful differences in the rates of occurrence for these relatively rare events. This may potentially explain the marked difference in only transfusion events.

\section{The Rates of Blood Transfusion Differed Between KID and Peds NSQIP}

The reported rates of blood transfusion after craniosynostosis surgery in the published literature varied between $1.7 \%$ and $100 \%$, typically as a function of the specific surgical technique and medical institution; series with predominantly endoscopic approaches generally reported transfusion rates less than $20 \%,{ }^{7-9,21}$ while series with predominantly open cranial vault remodeling cases generally reported transfusion rates greater than $80 \% .^{10,21,24}$ Variations in blood transfusion in the perioperative period after craniosynostosis surgery may reflect differences in surgical technique, institutional thresholds for transfusion, and type of synostosis requiring treatment. The transfusion rates that were reported in KID (36\%) were very different from those in Peds NSQIP (64\%). This discrepancy may reflect different surgical cohorts, with coding by CPT or

TABLE 3. Identification of complications

\begin{tabular}{lll}
\hline \multicolumn{1}{c}{ Complication } & \multicolumn{1}{c}{ KID (ICD-9 code) } & \multicolumn{1}{c}{ Peds NSQIP (clinical variable) } \\
\hline Infection & $\begin{array}{c}320.0-322.9,324.0-324.9,998.5,998.59,682.0,682.8- \\
682.9,686.8-686.9,410.0-411.9,411,412,417,418,419,\end{array}$ & $\begin{array}{c}\text { Superficial incisional surgical site } \\
\text { infection, deep incisional surgical site } \\
\text { infection }\end{array}$ \\
\hline Wound disruption & $930.00,730.08-730.09,730.90,730.98-730.99$ & Wound disruption \\
\hline Seizures & $780.3,780.39$ & Seizure occurrence \\
\hline Cardiac complication & 997.1 & $\begin{array}{c}\text { Cardiac arrest requiring cardiopulmo- } \\
\text { nary resuscitation }\end{array}$ \\
\hline $\begin{array}{l}\text { Stroke/intracranial } \\
\text { hemorrhage }\end{array}$ & $998.1,998.11-998.12,432.1$ & $\begin{array}{c}\text { Cerebrovascular accident/stroke or } \\
\text { intracranial hemorrhage }\end{array}$ \\
\hline Blood transfusion & $990.1-990.7$ & Occurrence of transfusion \\
\hline Sepsis & $381.0-384.9,380-389,995.90-995.94$ & Occurrence of sepsis \\
\hline
\end{tabular}


ICD-9-CM codes limiting our ability to distinguish open versus endoscopic techniques or type of craniosynostosis. There are additional potential explanations for this discrepancy: differences in surgical technique may be one. The underlying differences in patient population may be another. KID is designed to reflect a representative national sample, while Peds NSQIP data originates from a smaller number of hospitals that are not representative of the country. There may be a difference in patient age: age data in months is not available in this iteration of KID in 2012, and thus the mean age of surgery in months is not known. Although neither cohort definition sought to include syndromic children, there may be unaccounted differences in comorbid conditions or baseline health between the 2 data sets. KID may be underreporting transfusion events, either from omission or commission errors in coding. Peds NSQIP detects a higher rate of blood transfusions, and it is intuitive to accept that it is more likely to detect this event by clinical chart review rather than by administrative codes. However, further direct cross-validation studies are needed.

\section{Limitations}

Both data sources have limitations that are inherent to their focused time frames of analysis, as mentioned above. In the case of any surgery, especially craniosynostosis surgery, long-term outcomes are essential to understanding the success and sequelae. Neurocognitive, aesthetic, and patient/family satisfaction outcomes are notably not available in either database. No established international medical coding practice is in place to capture this information.

There are notable limitations to the Peds NSQIP data set, which includes limited details on diagnosis and clinical circumstances. Triggers for transfusion are not standardized. Operative indications, the locations of the affected sutures, and specific techniques are not known; the clinical significance of craniosynostosis data recorded in Peds NSQIP falls short because only CPT codes are used. By using CPT codes alone, the type and nature of craniosynostosis is not known, which is vital to understanding perioperative planning. The recorded outcomes are limited to 30-day follow-up. Although the data collection process for Peds NSQIP is rigorous, various patient and clinical details cannot be discerned. Anatomy and imaging characteristics are not available. Because patients and procedures were selected for this cohort using CPT procedure codes, we are cognizant that miscoding may be present, though the method of medical record review by trained Peds NSQIP staff may mitigate this possibility. Outcomes and health care utilization beyond 30 days are not captured here. Finally, this study is subject to the inherent biases of retrospective review. Also, the sampling method dictates that the year is divided into 468 8-day collection cycles, and only the first number of cases that met the inclusion criteria are captured. ${ }^{15}$ This method may not fully reflect the effects of a center's patient volume.

The neurosurgical literature demonstrates variable findings regarding the fidelity of data within large administrative data sets and the sensitivity and specificity of ICD-9 codes in capturing neurosurgical procedures. ${ }^{1,2,5,22,25}$ These limitations are inclusive of KID and include a lack of high granularity, clinically meaningful information about preoperative deformity type, anthropometric measurements, associated comorbidities, surgeon preferences, surgical techniques, and other factors that may have influenced the rate of complications. Overall disease complexity cannot be ascertained, and thus may confound some results. Because KID provides data on discrete hospitalizations, not individual patients, it is not possible to follow individual patients over time or distinguish between the first and revision procedures. The specific surgical technique cannot be ascertained using KID, and so the distinction between endoscopic suture release and the various subtypes of cranial vault remodeling cannot be clearly defined; the utilization of associated devices such as postoperative helmets or spring distractors is also not known. Furthermore, multicenter validation of ICD-9-CM coding with CPT coding will be needed to ensure that analyses capture similar surgical cohorts.

This project serves as the basis for a research proposal to access the multicenter Perioperative Craniofacial Surgery Prospective Registry (PCSPR), the anesthesiologyled prospective data collection of the Pediatric Craniofacial Collaborative Group (PCCG), which now totals over 20 centers, and has been ongoing for several years. At the time of the proposal to use PCSPR as the clinical standard to compare KID and NSQIP data, there were more than 1300 surgical cases collected with data collection overseen by the principal anesthesiologist investigator at each site. Cross-referencing administrative data, NSQIP data, and clinical registry data will be an important step to deepening the understanding of the relative strengths and limitations of each data set for further clinical research and development. This concept will be tested in pediatric craniofacial surgery, but may have larger implications for big data research.

\section{Conclusions}

This study reported the rate of perioperative complications after surgical intervention in children younger than 12 months with nonsyndromic craniosynostosis in 2012 by comparing 2 large data sources: a large administrative claims database (KID) and a clinical registry (Peds NSQIP). The rates of infection, wound disruption, seizures, cardiac events, and intracranial bleeds were low and similar between the 2 data sets, while the rates of blood transfusion were high and demonstrated a 2-fold discrepancy between the 2 data sources. Peds NSQIP identified twice as many blood transfusion events as KID. Focused understanding and further clinical validation are needed to appropriately interpret and harness the power of large data sets in order to measure and drive clinical quality.

\section{Acknowledgments}

American College of Surgeons National Surgical Quality Improvement Program and the hospitals participating in the ACS NSQIP are the source of the data used herein; they have not verified and are not responsible for the statistical validity of the data analysis or the conclusions derived by the authors. 


\section{References}

1. Berman MF, Stapf C, Sciacca RR, Young WL: Use of ICD-9 coding for estimating the occurrence of cerebrovascular malformations. AJNR Am J Neuroradiol 23:700-705, 2002

2. Carroll CP, Cochran JA, Guse CE, Wang MC: Are we underestimating the burden of traumatic brain injury? Surveillance of severe traumatic brain injury using centers for disease control International classification of disease, ninth revision, clinical modification, traumatic brain injury codes. Neurosurgery 71:1064-1070, 2012

3. Davenport DL, Holsapple CW, Conigliaro J: Assessing surgical quality using administrative and clinical data sets: a direct comparison of the University HealthSystem Consortium Clinical Database and the National Surgical Quality Improvement Program data set. Am J Med Qual 24:395-402, 2009

4. Ferreira MP, Collares MV, Ferreira NP, Kraemer JL, Pereira Filho Ade A, Pereira Filho Gde A: Early surgical treatment of nonsyndromic craniosynostosis. Surg Neurol 65 (Suppl 1):S22-S26, 2006

5. Greenberg JK, Ladner TR, Olsen MA, Shannon CN, Liu J, Yarbrough CK, et al: Validation of an International Classification of Diseases, Ninth Revision Code Algorithm for Identifying Chiari Malformation Type 1 Surgery in Adults. Neurosurgery 77:269-273, 2015

6. Harris DA, Lam S: Venous thromboembolism in the setting of pediatric traumatic brain injury. J Neurosurg Pediatr 13:448-455, 2014

7. Honeycutt JH: Endoscopic-assisted craniosynostosis surgery. Semin Plast Surg 28:144-149, 2014

8. Jimenez DF, Barone CM: Early treatment of coronal synostosis with endoscopy-assisted craniectomy and postoperative cranial orthosis therapy: 16-year experience. J Neurosurg Pediatr 12:207-219, 2013

9. Jimenez DF, Barone CM: Endoscopic technique for sagittal synostosis. Childs Nerv Syst 28:1333-1339, 2012

10. Lee HQ, Hutson JM, Wray AC, Lo PA, Chong DK, Holmes $\mathrm{AD}$, et al: Analysis of morbidity and mortality in surgical management of craniosynostosis. J Craniofac Surg 23:1256-1261, 2012

11. Lin Y, Pan IW, Harris DA, Luerssen TG, Lam S: The impact of insurance, race, and ethnicity on age at surgical intervention among children with nonsyndromic craniosynostosis. J Pediatr 166:1289-1296, 2015

12. McCarthy JG, Warren SM, Bernstein J, Burnett W, Cunningham ML, Edmond JC, et al : Parameters of care for craniosynostosis. Cleft Palate Craniofac J 49 Suppl:1S-24S, 2012

13. Nguyen C, Hernandez-Boussard T, Khosla RK, Curtin CM: A national study on craniosynostosis surgical repair. Cleft Palate Craniofac J 50:555-560, 2013

14. Pearson GD, Havlik RJ, Eppley B, Nykiel M, Sadove AM: Craniosynostosis: a single institution's outcome assessment from surgical reconstruction. J Craniofac Surg 19:65-71, 2008

15. Raval MV, Dillon PW, Bruny JL, Ko CY, Hall BL, Moss RL, et al: Pediatric American College of Surgeons National Surgical Quality Improvement Program: feasibility of a novel, prospective assessment of surgical outcomes. J Pediatr Surg 46:115-121, 2011

16. Sellers MM, Merkow RP, Halverson A, Hinami K, Kelz RR, Bentrem DJ, et al: Validation of new readmission data in the
American College of Surgeons National Surgical Quality Improvement Program. J Am Coll Surg 216:420-427, 2013

17. Seruya M, Oh AK, Boyajian MJ, Posnick JC, Myseros JS, Yaun AL, et al: Long-term outcomes of primary craniofacial reconstruction for craniosynostosis: a 12-year experience. Plast Reconstr Surg 127:2397-2406, 2011

18. Souweidane MM: Editorial. Periodical shifts in the surgical correction of sagittal craniosynostosis. J Neurosurg Pediatr 15:347-348, 2015

19. Tahiri Y, Paliga JT, Wes AM, Whitaker LA, Bartlett SP, Taylor JA: Perioperative complications associated with intracranial procedures in patients with nonsyndromic single-suture craniosynostosis. J Craniofac Surg 26:118-123, 2015

20. Thomas GP, Johnson D, Byren JC, Judge AD, Jayamohan J, Magdum SA, et al: The incidence of raised intracranial pressure in nonsyndromic sagittal craniosynostosis following primary surgery. J Neurosurg Pediatr 15:350-360, 2015

21. Vogel TW, Woo AS, Kane AA, Patel KB, Naidoo SD, Smyth MD: A comparison of costs associated with endoscope-assisted craniectomy versus open cranial vault repair for infants with sagittal synostosis. J Neurosurg Pediatr 13:324-331, 2014

22. Wang MC, Laud PW, Macias M, Nattinger AB: Strengths and limitations of International Classification of Disease Ninth Revision Clinical Modification codes in defining cervical spine surgery. Spine (Phila Pa 1976) 36:E38-E44, 2011

23. Warren SM, Proctor MR, Bartlett SP, Blount JP, Buchman SR, Burnett W, et al: Parameters of care for craniosynostosis: craniofacial and neurologic surgery perspectives. Plast Reconstr Surg 129:731-737, 2012

24. Wes AM, Paliga JT, Goldstein JA, Whitaker LA, Bartlett SP, Taylor JA: An evaluation of complications, revisions, and long-term aesthetic outcomes in nonsyndromic metopic craniosynostosis. Plast Reconstr Surg 133:1453-1464, 2014

25. Woodworth GF, Baird CJ, Garces-Ambrossi G, Tonascia J, Tamargo RJ: Inaccuracy of the administrative database: comparative analysis of two databases for the diagnosis and treatment of intracranial aneurysms. Neurosurgery 65:251-257, 2009

\section{Disclosures}

The authors report no conflict of interest concerning the materials or methods used in this study or the findings specified in this paper.

\section{Author Contributions}

Conception and design: Lam. Acquisition of data: Lam, Lin. Analysis and interpretation of data: Lam, Lin, Pan. Drafting the article: Lam, Lin. Critically revising the article: Lam, Pan, Mayer. Reviewed submitted version of manuscript: all authors. Approved the final version of the manuscript on behalf of all authors: Lam. Statistical analysis: Lin, Pan. Administrative/technical/material support: Lam, Pan. Study supervision: Lam.

\section{Correspondence}

Sandi Lam, Department of Neurosurgery, Texas Children's Hospital, 6701 Fannin St., CCC 1230.01, Houston, TX 77098. email: sklam@texaschildrens.org. 